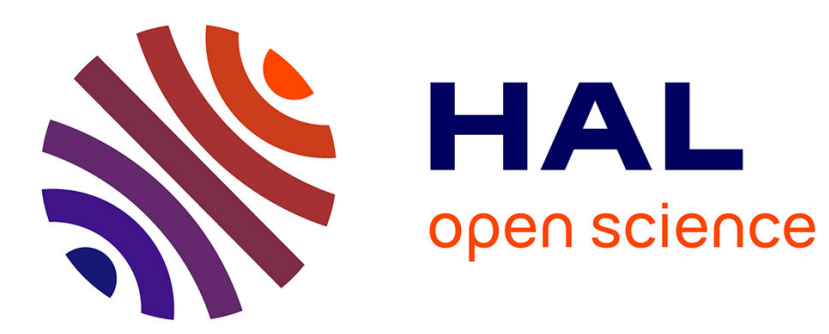

\title{
Shape Transition in Artificial Tumors: From Smooth Buckles to Singular Creases
}

Julien Dervaux, Yves Couder, Marie-Alice Guedeau-Boudeville, Martine Ben Amar

\section{- To cite this version:}

Julien Dervaux, Yves Couder, Marie-Alice Guedeau-Boudeville, Martine Ben Amar. Shape Transition in Artificial Tumors: From Smooth Buckles to Singular Creases. Physical Review Letters, 2011, 107 (1), 10.1103/PhysRevLett.107.018103 . hal-02326542

\section{HAL Id: hal-02326542 \\ https://hal.science/hal-02326542}

Submitted on 22 Oct 2019

HAL is a multi-disciplinary open access archive for the deposit and dissemination of scientific research documents, whether they are published or not. The documents may come from teaching and research institutions in France or abroad, or from public or private research centers.
L'archive ouverte pluridisciplinaire HAL, est destinée au dépôt et à la diffusion de documents scientifiques de niveau recherche, publiés ou non, émanant des établissements d'enseignement et de recherche français ou étrangers, des laboratoires publics ou privés. 


\title{
Shape Transition in Artificial Tumors: From Smooth Buckles to Singular Creases
}

\author{
Julien Dervaux, ${ }^{1}$ Yves Couder, ${ }^{2}$ Marie-Alice Guedeau-Boudeville, ${ }^{2}$ and Martine Ben Amar $^{1}$ \\ ${ }^{1}$ Laboratoire de Physique Statistique, Ecole Normale Supérieure, 24 rue Lhomond, 75005 Paris, France \\ ${ }^{2}$ Laboratoire Matière et Systèmes Complexes, Université Paris 7 Denis Diderot, 10 rue Alice Donon et Léonie Duquet, \\ 75013 Paris, France
}

(Received 19 February 2011; revised manuscript received 2 June 2011; published 1 July 2011)

\begin{abstract}
Using swelling hydrogels, we study the evolution of a thin circular artificial tumor whose growth is confined at the periphery. When the volume of the outer proliferative ring increases, the tumor loses its initial symmetry and bifurcates towards an oscillatory shape. Depending on the geometrical and elastic parameters, we observe either a smooth large-wavelength undulation of the swelling layer or the formation of sharp creases at the free boundary. Our experimental results as well as previous observations from other studies are in very good agreement with a nonlinear poroelastic model.
\end{abstract}

Heterogeneous or anisotropic growth is a known source of spontaneous deformations in both biological and inanimate matter [1-4]. Homogeneous volume variations can also induce nontrivial shapes if growing bodies are constrained in space [5]. Layered tissues provide a generic example of this process. Because they are structurally heterogeneous, distinct layers may grow at different rates, creating stresses which in turn can deform the system. Besides its importance for human-made systems [6], the buckling of layered structures is now thought to be involved in the morphogenesis of biological tissues such as fingerprints [7], brain convolutions [8], placodes [9], spheroidal fruits [10], or mucosa [11]. Many solid tumors also develop a layered structure during their avascular evolution. Nutrient depletion inside the tumor, due to the consumption at the margins, leads to the formation of a hypoxic core surrounded by a ring of highly proliferative cells [12]. This stratification slows the expansion of the tumor that eventually reaches a limiting size. To overcome this limitation, some tumors such as melanoma or glioblastoma lose their initial symmetry to further invade the surrounding tissues [13]. Although many biochemical processes underlie tumor development, one may wonder whether differential growth could contribute, by a buckling mechanism, to their morphological evolution.

In order to study the growth of layered tissues, we use hydrogels as highly monitorable abiotic substitutes for biological tissues. Besides their similar mechanical properties, some hydrogels can swell when immersed in a solvent. In this work, we exploit this swelling ability to mimic soft tissue growth and discuss the advantages and drawbacks of such an approach. Motivated by the geometry of melanoma, we investigate experimentally and theoretically the plane strain growth of a ring bound on one side to an elastic disk and free of traction on its other boundary as illustrated in Fig. 1.

The central part of the system is made of a neutral gel, swelling by at most $3 \%$ when immersed in water, while the external part is a ring of charged gel that swells by at least $300 \%$ in the same environment due to charges that favor straight configurations of the polymer chains. Swelling hydrogels are prepared as in [14] using the following protocol. For $1 \mathrm{ml}$ of pregel solution, $100 \mathrm{mg}$ of a mixture of neutral monomer (acrylamide), charged monomer (sodium acrylate), and cross-linker $\left(N, N^{\prime}\right.$-methylenebisacrylamide) is dissolved in distilled water. Nongrowing gels are prepared in the same fashion, although they do not contain any charged monomer. The gelification is initiated using $0.5 \mathrm{mg}$ of ammonium persulfate and catalyzed with $1 \mu \mathrm{l}$ of tetramethylenediamine (10\% wt/vol). First, a disk of neutral hydrogel is polymerized in a $1 \mathrm{~mm}$ thick circular mold of diameter $50 \mathrm{~mm}$. After polymerization, an external annulus of variable width is removed and replaced with the charged pregel solution, colored with turnsole (blue-violet). The tunable parameters of the system are (i) the elastic stiffness of the neutral and charged hydrogels, respectively $\mu_{I}$ and $\mu_{I I}$, which are proportional to the concentration of cross-linkers $[15,16]$ and (ii) the radius $A$ of the inner disk and the thickness $H$ of
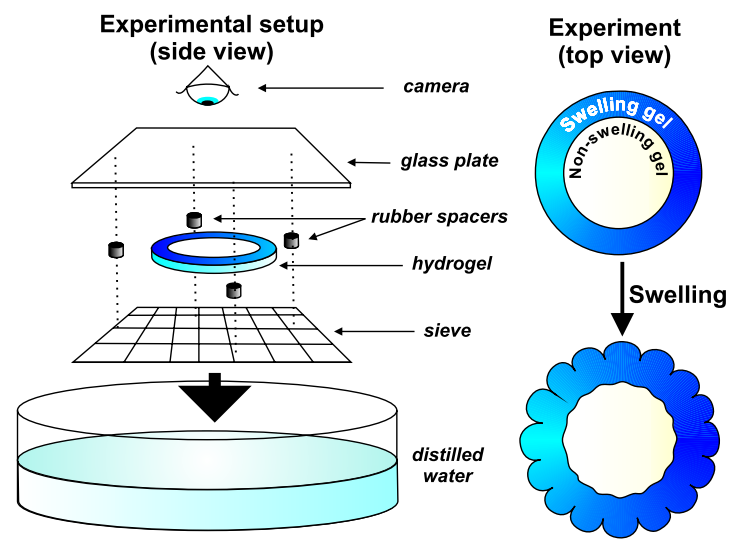

FIG. 1 (color online). Experimental setup (left) and schematic representation of the experiment (right). 
the growing ring. The system is then placed between a glass plate and a sieve (pore size $80 \mu \mathrm{m}$ ), with rubber spacers to control the gap, and immersed within distilled water for a few hours. Images are taken every 2 min.

In the following, we explore the evolution of the system in the phase space defined by the two dimensionless ratios $\mu_{I} / \mu_{I I}$ and $H / A$. The system first evolves from the reference configuration [Figs. 2(a) and 2(e)] in an axially symmetric fashion [Figs. 2(b) and 2(f)], then bifurcates to an oscillatory shape above a threshold in volume increase (depending on the geometrical and elastic parameters). We observe two distinct structures: for $\mu_{I} / \mu_{I I} \lesssim 1 \mathrm{a}$ buckling pattern appears, both boundaries of the annulus being nearly parallel in the vicinity of the instability threshold [Fig. 2(c)]. Further solvent uptake brings the outer edge of the oscillating ribbon in contact with itself and further breaks the symmetry. Conversely, for $\mu_{I} / \mu_{I I} \gtrsim 1$, the instability is entirely condensed in the ring with the formation of petals isolated by sharp creases while the inner interface disk-annulus remains approximately circular [Fig. 2(g)]. Such creases are reminiscent of the folds observed by Tanaka et al. [17,18]. In both sequences, the system ultimately breaks [Figs. 2(d) and 2(h)]. Additionally, we observe a color gradient in the radial direction, indicating an inhomogeneous water concentration, although the gel is everywhere in contact with the solvent. This distribution persists after several days, even though the volume has reached its final value corresponding to the thermodynamic equilibrium. To further quantify the instability, we have reported in Fig. 3(a) the dependence of the wavelength $\lambda$ on the thickness $H$ of the growing layer in the buckling $\left(\mu_{I} / \mu_{I I}=0.25\right)$ and creasing regimes $\left(\mu_{I} / \mu_{I I}=6\right)$. Those measurements clearly identify $H$ as the relevant length scale for the instability. Note that for $\mu_{I} / \mu_{I I} \leq 1$ and $H / A \geq 0.3$, the system does not exhibit the buckling instability but rather deforms in the transverse (thin) dimension. The central disk is stretched, becomes thinner, and its diameter increases. This effect is more pronounced when the core of the tumor is very soft. This three-dimensional effect might explain

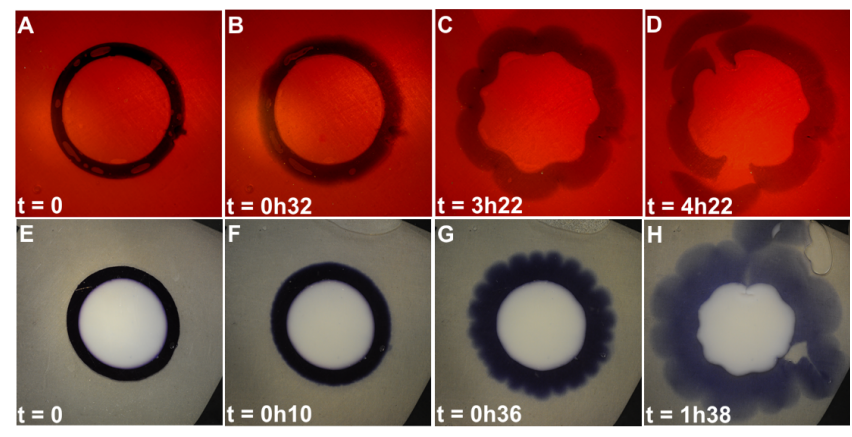

FIG. 2 (color online). Typical sequences of evolution for two systems with similar initial aspect ratio $[H / A \sim 0.26$ and 0.27 for sequence (a)-(d) and (e)-(h), respectively]. $\mu_{I} / \mu_{I I}$ is, respectively, 0.5 and 6 for sequences (a)-(d) and (e)-(h). In sequence (a)-(d), the water is colored with eosin. the relatively poor reproducibility of the experiments in this regime.

To understand how the elastic ratio $\mu_{I} / \mu_{I I}$ controls the instability, we record in Fig. 3(b) the wavelength $\lambda$, scaled by $H$, as a function of $\mu_{I} / \mu_{I I}$, clearly showing a transition between the two regimes around $\mu_{I} / \mu_{I I} \sim 1$. In the buckling regime, $\lambda$ results from a competition between the elasticity of the swelling ring and that of the inner disk, decreasing with increasing $\mu_{I} / \mu_{I I}$. When the wavelength (and thus the penetration length) falls below the ring thickness at threshold, the swelling rim and the disk uncouple and the system enters in a regime where $\lambda / H$ is of order 1 and independent of $\mu_{I} / \mu_{I I}$.

In order to explain quantitatively those results, we use a nonlinear poroelastic theory due to Gibbs and recently specialized to gels $[19,20]$. We consider a swelling elastic ring of gel (subscript $I I$ ) surrounding an incompressible disk (subscript $I$ ). The position of a point in the reference (current) configuration is $\vec{R}(\vec{r})$ and $\mathbf{F}=\partial \vec{r} / \partial \vec{R}$ describes the local deformation. In cylindrical coordinates, bodies $I$ and $I I$ occupy the regions $0 \leq R \leq A, 0 \leq \Theta \leq 2 \pi$ and $A \leq R \leq B \equiv A+H, 0 \leq \Theta \leq 2 \pi$. The gel is a network of cross-linked polymer chains associated with a solvent at the concentration $C(\vec{R})$. The system is in contact with a solvent reservoir held at chemical potential $\mu$ so the solvent can enter or leave the network. Physically, $\mu$ represents the energetical cost of exchanging a solvent particle between the gel and the reservoir. At the thermodynamic equilibrium, the chemical potential in the gel is constant and equal to $\mu$ and the grand potential of the gel $\hat{\mathcal{W}}_{I I}(\mathbf{F}, \mu)=\mathcal{W}_{I I}(\mathbf{F}, C)-\mu C$ is minimized, $\mathcal{W}_{I I}$ being its Helmholtz free energy. The $C$ dependence can be eliminated by assuming the molecular constituents of the

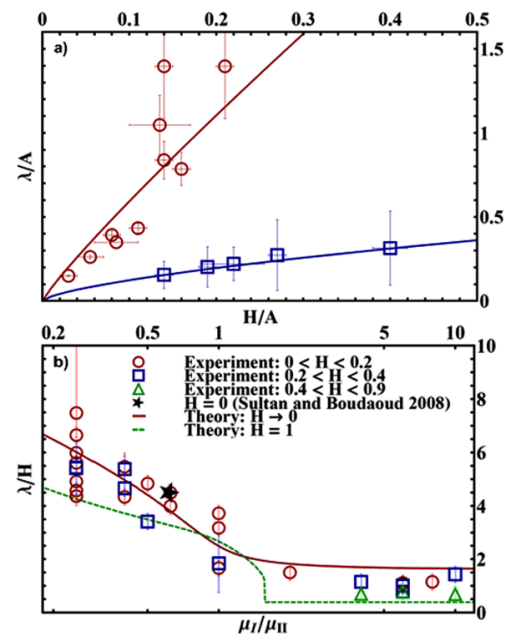

FIG. 3 (color online). (a) Wavelength $\lambda$ as a function of the initial thickness $H$ for $\mu_{I} / \mu_{I I}=0.25$ (red circles) and $\mu_{I} / \mu_{I I}=6$ (blue squares). The lengths are normalized by the inner radius $A$. (b) Reduced wavelength $\lambda / H$ as a function of $\mu_{I} / \mu_{I I}$ for various thickness. Experimental results from [25] are shown. The lines are the theoretical predictions. 
network to be incompressible, i.e., $1+v C=\operatorname{det} \mathbf{F} \equiv J$, where $v C$ is the volume of solvent divided by the volume of the dry network [19]. From the statistical theory of polymer chains [15], we choose the simplest possible form for $\mathcal{W}_{I I}=\mu_{I I} / 2\left(\lambda_{I I r}^{2}+\lambda_{I I \theta}^{2}-2-2 \log J_{I I}\right)$, where $\lambda_{I I r}^{2}$ and $\lambda_{I I \theta}^{2}$ are the eigenvalues of $\mathbf{F}^{\mathrm{T}} \mathbf{F}$. The equilibrium condition reads DivS $\mathbf{S}_{I I}=\overrightarrow{0}$, where $\mathbf{S}_{I I}=\partial \hat{\mathcal{W}}_{I I} / \partial \mathbf{F}$ is the nominal stress. There is also a surface energy associated with the gel-water interface and thus $\mathbf{S}_{I I}^{\mathrm{T}} \vec{N}=$ $-\sigma k J_{I I} \mathbf{F}^{-\mathrm{T}} \vec{N}$ on this boundary, where $\sigma, k$, and $\vec{N}$ are, respectively, the surface energy, curvature, and outer unit normal vector associated with the free boundary. At short time, the system evolves in an axially symmetric fashion. Because the disk is incompressible, it is mapped to the region $0 \leq r_{I}(R)=R \leq r_{I}(A)=A, \quad 0 \leq \theta=\Theta \leq 2 \pi$, while the annulus is mapped to $A \leq r_{I I}(R) \leq r_{I I}(B)=$ $b \equiv A+h, 0 \leq \theta=\Theta \leq 2 \pi$. The inner disk being unstretched in this nonlinear configuration, only its linear properties are relevant for the stability analysis and, under the assumptions of material isotropy, homogeneity and incompressibility, it is characterized by a single elastic constant $\mu_{I}$ (the infinitesimal shear modulus). The only nonvanishing equilibrium equation in the ring is $r_{I I}^{\prime \prime}[1+$ $\left.\left(r_{I I}^{\prime}\right)^{-2}\right]+r_{I I}^{\prime} / R\left[1-\left(r_{I I}^{\prime}\right)^{-2}\right]-r_{I I} / R^{2}+1 / r_{I I}=0, \quad$ with $r_{I I}(A)=A$ and $S_{I I r r}(B)=-\sigma / B$. This equation is integrated numerically. The ring is under circumferential compression and radial tension while the dilatation $J_{I I}$ (and hence $C$ ) increases from the inner to the free boundary. This heterogeneity results from the inhibition of swelling by the stresses which are stronger near the core (Fig. 4). The radial and orthoradial components of the stress $\mathbf{S}_{I}$ in the disk are given by the continuity of normal traction, yielding $\quad S_{I r r}=S_{I \theta \theta}=-\mu / v-\mu_{I I}\left[1 / r^{\prime}(1)-r^{\prime}(1)\right]$, and the disk is thus under homogeneous isotropic tension. We now study the stability of this axially symmetric solution by adding to the base state an infinitesimal perturbation $\left[u^{(1)}(r) \cos (m \theta), v^{(1)}(r) \sin (m \theta)\right]$ and by linearizing the equilibrium and constitutive equations [21]. This yields the usual equations of linear elasticity with an apparent elastic modulus depending on the prestressed

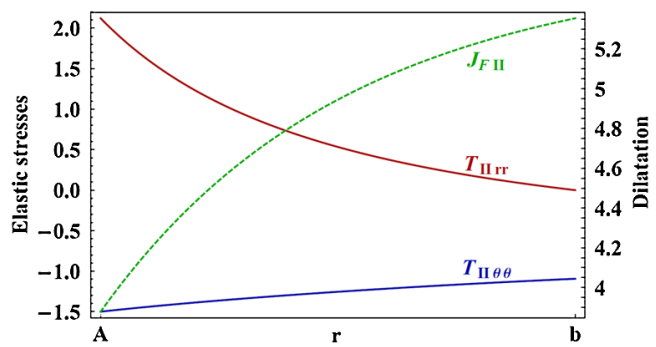

FIG. 4 (color online). Base state. Radial $\left(T_{I I r}\right.$ - red solid line) and circumferential $\left(T_{I I \theta \theta}\right.$-blue) solid line) components of the Cauchy (true) stress $\mathbf{T}_{I I}=J_{F I I}^{-1} \mathbf{F}_{I I} \mathbf{S}_{I I}$ together with the dilatation ( $J_{F I I}$ - green dashed line) of the ring as a function of the current space variable $r$. The physical parameters are $B / A=1.5$, $\mu_{I} / \mu_{I I}=1, \mu=1.5$, and $\sigma /\left(\mu_{I I} A\right)=10^{-4}$. configuration. Within this framework, swelling decreases the effective stiffness of the gel. In the present situation, because the stretches depend on space and orientation, so does the apparent Young's modulus, and the swollen ring behaves as an anisotropic, heterogeneous linear material in the vicinity of the base state [22]. Together with the two linear ordinary differential equations of second order for each media, we supply eight boundary conditions: the continuity of displacement at $r=A$ (two equations) and mechanical equilibrium at $r=A, b$ (four equations). In addition, physical quantities must stay bounded at $r=0$ (two equations). This boundary-value problem is solved numerically by fixing the parameters $B / A, \mu_{I} / \mu_{I I}$, and $\sigma /\left(\mu_{I I} A\right)$ and finding the wave number $m$ that minimizes the threshold thickness. Alternatively, we may take the wavelength $\lambda=2 \pi A / m$ as an order parameter which, for the sake of clarity, is taken as a continuous parameter. We take the value $\sigma=0.1 \mathrm{mN} / \mathrm{m}$ for the surface tension [23]. The results are shown as solid lines in Fig. 3 and are in excellent agreement with experimental measurements. Further analytical progress can be made in the limit $A \rightarrow \infty$ which corresponds to a planar swelling layer bound to a half-space [22]. We only record here the asymptotic results. For a very soft disk $\left(\mu_{I} / \mu_{I I} \ll 1\right)$ we find for the wavelength and thickness at threshold $h$ :

$$
\frac{\lambda}{H}=\frac{2 \pi}{6^{1 / 3}}\left(\frac{\mu_{I}}{\mu_{I I}}\right)^{-1 / 3} \text { and } \frac{h}{H}=1+\frac{3^{2 / 3}}{2^{4 / 3}}\left(\frac{\mu_{I}}{\mu_{I I}}\right)^{2 / 3} .
$$

A similar scaling law can be obtained for a layer with a prescribed volumetric strain on a compliant substrate although it predicts higher wavelengths and thresholds [24]. In opposition to biological growth, swelling does not involve solid matter creation but rather a migration of solvent that decreases the cross-linkers' density and hence the stiffness. Bifurcations thus occur at lower threshold, explaining recently reported discrepancies [22,25]. This distinction is even more striking in the hard disk limit $\left(\mu_{I} / \mu_{I I} \gg 1\right)$ where, following [5], we find [22]

$$
\begin{aligned}
& \frac{\lambda}{H} \sim 4 \pi / \log \left[\frac{44.95}{d}\right] \text { and } \\
& \frac{h}{H} \sim 1.51+0.45 d \log \left(\frac{44.95}{d}\right)
\end{aligned}
$$

under the assumption that $d=\sigma /\left(\mu_{I I} H\right)$ is small (at most $10^{-4}$ in our experiments). In agreement with the experimental observations, the wavelength is of order $H$, independent of $\mu_{I} / \mu_{I I}$, and depends weakly on $\sigma$. The surface tension prevents the wavelength to collapse to zero, a socalled surface instability, predicted for the related problem of an elastic half-space in compression [26]. By contrast, however, the swelling layer bifurcates when it reaches roughly 1.5 times its initial volume, a value much lower than its counterpart for biological growth or compression $(\sim 3$ times the initial value [5]), in agreement with our findings and previous results [14]. 


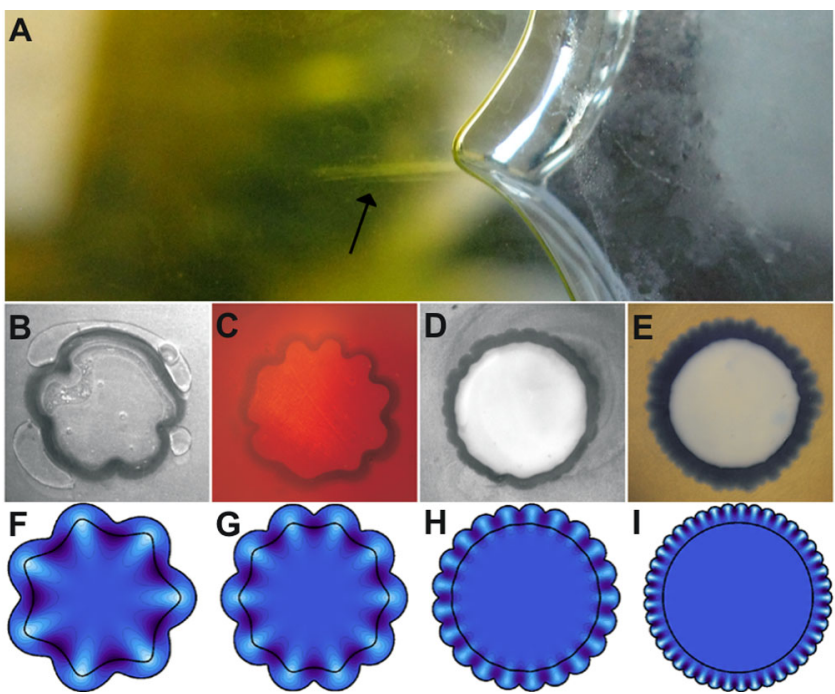

FIG. 5 (color online). (a) Close-up view of the cusped structure. Although the system is out of the solvent bath, a thin layer of water wets the free boundary which appears smooth. The arrow indicates an opaque region below the cusp which might be associated with a spinodal decomposition. (b)-(e) Various experiments with similar aspect ratios $H / A \sim 0.13$ and increasing values of $\mu_{I} / \mu_{I I}$ (from left to right: $0.25,0.5,1,6$ ). (f)(i) Corresponding theoretical predictions. Although the absolute amplitude of the deformation is not known, the relative amplitude (between the inner and outer interfaces) shows a condensation of the deformation at the free boundary.

Although our analysis correctly describes the onset of this instability, its nonlinear evolution exhibits interesting features [27] that are not fully understood. Firstly, adjacent buckles quickly come in contact, greatly impacting the evolution of the system above the instability threshold. Moreover, the creasing structure possibly involves a spinodal decomposition of the gel due to the stress focusing at the junction between consecutive petals (Fig. 5). This phase separation might lock the system in a metastable state by creating an energy barrier preventing the reorganization of the solvent inside the network.

While gel swelling is a relaxation towards thermodynamic equilibrium, tumor growth is an active process. This distinction hinders further comparison between the dynamics of growth and swelling. On the other hand, by capturing the effects of geometry, nonlinear elasticity, and stressmodulated growth, our study shed light on the mechanics and morphogenesis of tumors. From a clinical standpoint, the contour shape of melanoma is highly relevant for diagnosis and indicates a shift of a benign nevus toward an aggressive behavior. The ABCD system (asymmetry, border irregularity, color, diameter) is a widely used visual criteria for assessing the potential malignancy of a skin lesion. The contour of the melanoma may indeed exhibit long (A) or short (B) wavelength undulations, as observed in our artificial tumor. Moreover, the thickness of the proliferative ring being fixed by the competition between diffusion and growth, a large diameter implies a small aspect ratio and thus, according to our model, a low bifurcation threshold. This is consistent with the use of the $\mathrm{D}$ criteria in melanoma diagnosis.

We have investigated the morphogenesis of layered tissues in a previously unexplored geometry and unraveled a transition between two qualitatively distinct regimes. Experimental results have been well described by a detailed nonlinear poroelastic model and scaling laws given for thin swelling rings. Our approach also provides a simple way to investigate in detail the challenging problem of stress-modulated growth. Finally, the present study clarifies the conditions for cusp formation, highly singular structures still resisting theoretical explanation.

[1] A. Goriely and M. Ben Amar, Phys. Rev. Lett. 94, 198103 (2005).

[2] J. Genzer and J. Groenewold, Soft Matter 2, 310 (2006).

[3] Y. Klein, E. Efrati, and E. Sharon, Science 315, 1116 (2007).

[4] J. Dervaux and M. Ben Amar, Phys. Rev. Lett. 101, 068101 (2008).

[5] M. Ben Amar and P. Ciarletta, J. Mech. Phys. Solids 58, 935 (2010).

[6] N. Bowden et al., Nature (London) 393, 146 (1998).

[7] M. Kücken and A.C. Newell, Europhys. Lett. 68, 141 (2004).

[8] D. Richman et al., Science 189, 18 (1975).

[9] J. Dervaux and M. Ben Amar, IMA J. Appl. Math. 75, 571 (2010).

[10] J. Yin et al., Proc. Natl. Acad. Sci. U.S.A. 105, 19132 (2008).

[11] B. Li et al., J. Mech. Phys. Solids 59, 758 (2011).

[12] W. Mueller-Klieser, Crit. Rev. Oncol./Hematol. 36, 123 (2000).

[13] H. Frieboes et al., Cancer Res. 66, 1597 (2006).

[14] V. Trujillo, J. Kim, and R. C. Hayward, Soft Matter 4, 564 (2008).

[15] P. J. Flory, Principles of Polymer Chemistry (Cornell University Press, Ithaca, NY, 1953).

[16] T. Yeung et al., Cell Motil. Cytoskeleton 60, 24 (2005).

[17] T. Tanaka, Physica (Amsterdam) 140A, 261 (1986).

[18] T. Tanaka et al., Nature (London) 325, 796 (1987).

[19] W. Hong et al., J. Mech. Phys. Solids 56, 1779 (2008).

[20] W. Hong, Z. Liu, and Z. Suo, Int. J. Solids Struct. 46, 3282 (2009).

[21] R. W. Ogden, Non-Linear Elastic Deformations (Dover, New York, 1997).

[22] J. Dervaux and M. Ben Amar, J. Mech. Phys. Solids 59, 538 (2011).

[23] R. N. King et al., J. Colloid Interface Sci. 103, 62 (1985).

[24] H. G. Allen, Analysis and Design of Structural Sandwich Panels (Pergamon, New York, 1969).

[25] E. Sultan and A. Boudaoud, J. Appl. Mech. 75, 051002 (2008).

[26] M. A. Biot, Appl. Sci. Res. Sect. A 12, 168 (1963).

[27] E. Hohlfeld and L. Mahadevan, Phys. Rev. Lett. 106, 105702 (2011). 